\title{
Electronic spectra and photodissociation of vinyl chloride: A symmetry-adapted cluster configuration interaction study
}

\author{
Sundaram Arulmozhiraja, Ryoichi Fukuda, and Masahiro Ehara \\ Department of Synthetic Chemistry and Biological Chemistry, Graduate School of Engineering, \\ Kyoto University, Katsura, Nishikyou-ku, Kyoto 615-8510, Japan \\ Hiroshi Nakatsuji ${ }^{\text {a) }}$ \\ Department of Synthetic Chemistry and Biological Chemistry, Graduate School of Engineering, \\ Kyoto University, Katsura, Nishikyou-ku, Kyoto 615-8510, Japan and Fukui Institute for Fundamental \\ Chemistry, Kyoto University, 34-4 Takano, Nishihiraki-cho, Sakyo-ku, Kyoto 606-8103, Japan
}

(Received 27 October 2005; accepted 7 December 2005; published online 20 January 2006)

The vertical absorption spectrum and photodissociation mechanism of vinyl chloride (VC) were studied by using symmetry-adapted cluster configuration interaction theory. The important vertical $\pi \rightarrow \pi^{*}$ excitation was intensively examined with various basis sets up to aug-cc-pVTZ augmented with appropriate Rydberg functions. The excitation energy for $\pi \rightarrow \pi^{*}$ transition obtained in the present study, $6.96 \mathrm{eV}$, agrees well with the experimental value, $6.7-6.9 \mathrm{eV}$. Calculated excitation energies along with the oscillator strengths clarify that the main excitation in $\mathrm{VC}$ is the $\pi \rightarrow \pi^{*}$ excitation. Contrary to the earlier theoretical reports, the results obtained here support that the $\mathrm{C}-\mathrm{Cl}$ bond dissociation takes place through the $n_{\mathrm{Cl}}-\sigma_{\mathrm{C}-\mathrm{Cl}}^{*}$ state. (C) 2006 American Institute of Physics.

[DOI: $10.1063 / 1.2162539]$

\section{INTRODUCTION}

Vinyl chloride $\left(\mathrm{C}_{2} \mathrm{H}_{3} \mathrm{Cl}\right)(\mathrm{VC})$ is an atmospheric pollutant. Evidence showed that $\mathrm{VC}$ causes cancer in human beings. Because of this, understanding of the photochemistry and spectroscopy of VC is important. There were many studies in this direction in the past; however, detailed studies to get a clear picture about the photochemical behavior of $\mathrm{VC}$ are still necessary, because the results obtained in some of the previous studies are ambiguous and speculative. Additionally, the conclusions made in a few recent studies are very much in contradiction with the existing predictions. For example, two recent theoretical studies ${ }^{1,2}$ assigned the $\pi$ - $3 \mathrm{~s}$ Rydberg state as the optically bright state. It is completely contradictory to the generally agreed fact that for ethylenic compounds the strongest absorption is due to $\pi \rightarrow \pi^{*}$ transition. ${ }^{3}$ Another fact is that the VC excited to the bright $\pi-\pi^{*}$ state has generally been thought to be predissociated through repulsive $n_{\mathrm{Cl}}-\sigma_{\mathrm{C}-\mathrm{Cl}}^{*}$ state. ${ }^{4,5}$ Though a reassignment to a $\pi-\sigma_{\mathrm{C}-\mathrm{Cl}}^{*}$ state has been recently suggested, ${ }^{6}$ it was promptly questioned lately. ${ }^{7}$ Surprisingly, while concluding that the dissociation of fast chlorine atoms occurs through the $\pi-\sigma_{\mathrm{C}-\mathrm{Cl}}^{*}$ state, the role of the $n_{\mathrm{Cl}} \sigma_{\mathrm{C}-\mathrm{Cl}}^{*}$ state in the dissociation was completely ignored in a recently published report. $^{2}$

Studies focusing on spectroscopy $\mathrm{y}^{3,4,8-18}$ and photodissociation mechanism ${ }^{4-7,19-24}$ of $\mathrm{VC}$ were made in the past. The bright peak at $6.7-6.9 \mathrm{eV}$ has been considered and agreed to be due to the $\pi \rightarrow \pi^{*}$ excitation. ${ }^{4,8,10-12}$ Umemoto et al. ${ }^{5}$ studied the dissociation mechanism of a few chloroethylenes including VC. They concluded that two processes are com-

\footnotetext{
${ }^{a)}$ Author to whom correspondence should be addressed. Electronic mail: hiroshi@sbchem.kyoto-u.ac.jp
}

peting: one is the transition to a dissociative $n_{\mathrm{Cl}} \sigma_{\mathrm{C}-\mathrm{Cl}}^{*}$ state which crosses the bright $\pi-\pi^{*}$ state in the $\mathrm{C}-\mathrm{Cl}$ coordinate and the other is the internal conversion to the ground state. The $\mathrm{C}-\mathrm{Cl}$ bond cleavage resulted in the former process and $\mathrm{HCl}$ elimination occurred in the latter. Umemoto et al. ${ }^{5}$ speculated that twisting of the $\mathrm{C}=\mathrm{C}$ bond leads to mixing of the $\pi-\pi^{*}$ and $\pi-\sigma_{\mathrm{C}-\mathrm{Cl}}^{*}$ states. Internal conversion to the $\pi-\sigma_{\mathrm{C}-\mathrm{Cl}}^{*}$ state may serve as intermediate state from which internal conversion to the ground state occurs. It is obvious that the $\pi-\sigma_{\mathrm{C}-\mathrm{Cl}}^{*}$ state was considered as a bound state in that study. However, Tonokura et al. ${ }^{6}$ later speculated the $\pi-\sigma_{\mathrm{C}-\mathrm{Cl}}^{*}$ state as a repulsive state. From their calculated vertical excitation energies, they claimed that $193 \mathrm{~nm}(6.42 \mathrm{eV})$ photoexcitation is not sufficient to reach the crossing point of the $\pi-\pi^{*}$ and $n_{\mathrm{Cl}}-\sigma_{\mathrm{C}-\mathrm{Cl}}^{*}$ states and hence they suggested that the $\pi-\sigma_{\mathrm{C}-\mathrm{Cl}}^{*}$ state is responsible for the $\mathrm{C}-\mathrm{Cl}$ bond breaking, but Blank et al. ${ }^{7}$ raised doubts over this assignment.

Chang and Chen ${ }^{1}$ studied few low-lying electronic states of VC by using complete active space self-consistent field (CASSCF) and multireference configuration interaction (MRCI) theories. Their calculated vertical excitation energies for the $\pi \rightarrow \pi^{*}$ transition which is well established as responsible for the bright peak in the absorption spectrum are around $0.6-0.8 \mathrm{eV}$ higher than the experimental values (strong peak). However, the authors assigned the $\pi-3 s$ Rydberg state as the optically bright state on the basis of their calculated adiabatic excitation energies. Through configuration interaction singles calculation, ${ }^{1}$ a long tail observed at $45000-54000 \mathrm{~cm}^{-1}(5.58-6.70 \mathrm{eV})$ in the absorption spectrum ${ }^{4}$ was speculated due to the $1^{3} A^{\prime \prime} \leftarrow 1{ }^{1} A^{\prime}$ and $2^{3} A^{\prime \prime} \leftarrow 1{ }^{1} A^{\prime}$ transitions via spin-orbit coupling. As a result, Chang and $\mathrm{Chen}^{1}$ also speculated that upon the excitation of $\mathrm{VC}$ at $193 \mathrm{~nm}(6.42 \mathrm{eV})$, the $1^{3} A^{\prime \prime}$ or $2^{3} A^{\prime \prime}$ excited state, 
instead of $\pi-\pi^{*}$, is initially prepared for the photodissociation processes. These speculations were contradictory to the existing reports. However, Chang ${ }^{2}$ studied triplet excited states of VC using CASSCF and MRCI and from the calculated values he abandoned the earlier speculations that (1) the second and third triplet excited states might be responsible for the absorption spectra at $45000-54000 \mathrm{~cm}^{-1}$ $(5.58-6.70 \mathrm{eV})$ and (2) the $1^{3} A^{\prime \prime}$ or $2^{3} A^{\prime \prime}$ state might involve in the photodissociation process. But, he maintains that the strong peak in the absorption spectra is due to the Rydberg $\pi \rightarrow 3 s$ excitation. Chang ${ }^{2}$ also studied the potentialenergy surface of the first singlet excited state of $\mathrm{VC}$ and concluded that the chlorine dissociation occurs via $\pi-\sigma_{\mathrm{C}-\mathrm{Cl}}^{*}$ state followed by a crossing between $\pi-3 s$ and $\pi$ - $\sigma_{\mathrm{C}-\mathrm{Cl}}^{*}$ states. These conclusions are inconsistent with the existing speculations.

In these circumstances, we made the present study on VC by using symmetry-adapted cluster ${ }^{25}$ /configuration interaction $^{26-28}$ (SAC-CI) theory to clear the existing contradictions. Considering the importance of the photochemistry of chloroethylenes, comprehensive and accurate knowledge about vinyl chloride, the smallest molecule in the chloroethylene's family, is vital. The main focus of this paper is twofold: (1) studying the electronic spectrum of VC by using SAC-CI to assign the excited states including the valence $\pi-\sigma_{\mathrm{C}-\mathrm{Cl}}^{*}, \pi-\pi^{*}, n_{\mathrm{Cl}}-\sigma_{\mathrm{C}-\mathrm{Cl}}^{*}, n_{\mathrm{Cl}^{-}} \pi^{*}$, and the Rydberg states and (2) understanding the $\mathrm{C}-\mathrm{Cl}$ bond breaking mechanism in $\mathrm{VC}$ by studying the potential-energy surfaces (PESs) of its excited states including the important $n_{\mathrm{Cl}^{-}} \sigma_{\mathrm{C}-\mathrm{Cl}}^{*}$ state.

\section{COMPUTATIONAL DETAILS}

All computations were performed with GAUSSIAN 03 suite of programs. ${ }^{29}$ The vertical absorption spectra were obtained using SAC-CI singles and doubles (SD)- $R$ method utilizing many different basis sets ranging from Dunning's double zeta $[\mathrm{D} 95(d, p)]$ (Ref. 30) to augmented triple zeta type (aug-cc-pVTZ). ${ }^{31}$ The influence of polarization and diffuse functions was explored. Notations, for example, cc-pVTZ $(-d)$ for $\mathrm{H}$ representing the basis set cc-pVTZ without $d$ polarization function on $\mathrm{H}$ atoms and aug-cc-pVTZ(- $f)$ for $\mathrm{C}$ and $\mathrm{Cl}$ atoms representing the basis set aug-cc-pVTZ without $f$ diffuse function on $\mathrm{C}$ and $\mathrm{Cl}$ atoms are used here. Calculations were made using these basis sets which were augmented with two sets of Rydberg spd functions for carbon $\left(\zeta_{s}=0.0437\right.$ and $0.01725, \zeta_{p}$ $=0.0399$ and 0.01575 , and $\zeta_{d}=0.0285$ and 0.01125 ) and two sets for chlorine $\left(\zeta_{s}=0.0475\right.$ and $0.01875, \zeta_{p}=0.0380$ and 0.0150 , and $\zeta_{d}=0.0285$ and 0.01125$)$. Chlorine Rydberg functions were placed on chlorine atom in all the calculations while carbon Rydberg functions were used in two ways: placing the functions (1) on both carbons and (2) on $\mathrm{C}=\mathrm{C}$ bond center (BC). The preliminary PES of several low-lying excited states along the $\mathrm{C}-\mathrm{Cl}$ bond and $\mathrm{C}=\mathrm{CH}_{2}$ twisting coordinates were obtained mainly by using $\mathrm{D} 95(d, p)$ basis set in this study. Electronic spectra calculations were done within $C_{s}$ symmetry, with the molecule placed in the $x y$ plane.

\section{RESULTS AND DISCUSSIONS}

Before going to the calculations on $\mathrm{VC}$, we studied $\pi$ $\rightarrow \pi^{*}$ transition of ethylene to examine the basis sets to study VC. Since VC is similar to ethylene and there were many theoretical studies on ethylene, such examination is meaningful. The strong peak in the excitation spectrum of ethylene is the broadband with the maximum at 7.65 (Ref. 32) or $7.66 \mathrm{eV},{ }^{33}$ which arises from the $\pi \rightarrow \pi^{*}$ transition. This broadband is overlapped by several Rydberg states. While the exact vertical excitation energy of this transition has not been obtained experimentally, high-level theoretical studies have converged to a value of $8 \mathrm{eV} .^{34}$ Finally, this energy has been estimated to fall in between 7.90 and $7.95 \mathrm{eV}$ on the basis of sophisticated theoretical calculations. ${ }^{35,36}$

The results obtained for the $\pi \rightarrow \pi^{*}$ transition of ethylene in the present study were tabulated in Table I. The table clearly shows that the SAC-CI excitation energies are very accurate and are in close agreement with the available theoretical results. Preliminary calculations were performed on VC to obtain the excitation energies for the $\pi \rightarrow \pi^{*}$ transition. The results were presented in Table II. These calculations conclude the following two points: (1) Though the results obtained by placing the Rydberg diffuse functions either on the carbon atoms or on the $\mathrm{C}=\mathrm{C}$ bond center do not have much differences, the former has a slight edge over the latter and (2) aug-cc-pVTZ basis set for heavy atoms seems very essential to obtain accurate energies, while cc-pVTZ basis set, even without $d$-type polarization function, is enough for hydrogen atoms. By keeping these points in mind, we performed the calculations of the vertical excitation spectrum in the wide energy region of $\mathrm{VC}$.

Table III shows the vertical excitation energies and oscillator strengths calculated using SAC-CI with LevelThree, along with the results obtained in the previous theoretical and experimental studies for the singlet excited states in each $\left(A^{\prime}\right.$ and $\left.A^{\prime \prime}\right)$ symmetry. The lowest excited state can be better denoted as $\pi-3 s / \sigma_{\mathrm{C}-\mathrm{Cl}}^{*}$ since a small contribution from $\pi$ $\rightarrow \sigma_{\mathrm{C}-\mathrm{Cl}}^{*}$ transition is also involved in this Rydberg excitation.

The second excited state represents $\pi \rightarrow \pi^{*}$ excitation. The excitation energy, $6.96 \mathrm{eV}$, obtained for this transition is close to the experimental values, $6.7-6.9 \mathrm{eV}$. On the other hand, the values derived from MRCI (Refs. 1 and 6) were $0.5-0.76 \mathrm{eV}$ higher than the experimental values. Tonokura et al. ${ }^{6}$ used cc-pVTZ basis set with a set of $s$ and $p$ diffuse functions on chlorine in their MRCI calculations, while Chang and $\mathrm{Chen}^{1}$ adopted $6-311+\mathrm{G}(d, p)$ basis set with a diffuse $s p$ function on each carbon. The present calculations reveal that the basis sets used in those two MRCI studies were not sufficient to properly describe $\pi \rightarrow \pi^{*}$ excitations. Using the basis set by Chang and Chen, ${ }^{1}$ we performed the SAC-CI calculations on ethylene and VC. The excitation energy for $\pi \rightarrow \pi^{*}$ transition in VC was calculated to be $7.32 \mathrm{eV}$. Though this value is better than that of MRCI with the same basis set $(7.46 \mathrm{eV})$, it is far from our best SAC-CI value $(6.96 \mathrm{eV})$. On the basis of their calculation, Chang and Chen ${ }^{1}$ assigned the maximum at $6.71 \mathrm{eV}$ in the absorption spectrum is due to the $\pi \rightarrow 3 s$ Rydberg transition. They proposed that the observed peaks at 6.82 and $6.88 \mathrm{eV}$ are pos- 
TABLE I. Ethylene $\pi \rightarrow \pi^{*}$ vertical excitation energies ( $\Delta E$ in $\left.\mathrm{eV}\right)$ using the SAC-CI method with various basis sets at experimental geometry (Ref. 37).

\begin{tabular}{|c|c|c|c|c|}
\hline \multirow[b]{2}{*}{ Method } & \multicolumn{2}{|c|}{ Basis set } & \multirow{2}{*}{$\begin{array}{l}\text { Rydberg function } \\
\text { (two sets of } s p d \text { ) }\end{array}$} & \multirow[b]{2}{*}{$\Delta E$} \\
\hline & $\mathrm{H}$ & $\mathrm{C}$ & & \\
\hline \multirow{11}{*}{ SAC-CI } & DZP & DZP & On BC & 8.195 \\
\hline & cc-pVTZ & cc-pVTZ & On BC & 8.027 \\
\hline & cc-pVTZ & aug-cc-pVTZ & On BC & 7.949 \\
\hline & cc-pVTZ & aug-cc-pVQZ & On BC & 7.922 \\
\hline & DZP & DZP & On C & 8.142 \\
\hline & cc-pVTZ & cc-pVTZ & On $\mathrm{C}$ & 8.098 \\
\hline & cc-pVTZ(-pd) & aug-cc-pVTZ(-f) & On $\mathrm{C}$ & 8.029 \\
\hline & cc-pVTZ(-pd) & aug-cc-pVTZ & On $\mathrm{C}$ & 7.981 \\
\hline & cc-pVTZ(-d) & aug-cc-pVTZ & On $\mathrm{C}$ & 7.959 \\
\hline & cc-pVTZ & aug-cc-pVTZ(-f) & On $\mathrm{C}$ & 7.967 \\
\hline & cc-pVTZ & aug-cc-pVTZ & On $\mathrm{C}$ & 7.943 \\
\hline CASPT2 & & & & $8.00^{\mathrm{a}}$ \\
\hline Best theory estimate & & & & $7.90-7.95^{\mathrm{b}}$ \\
\hline Expt. & & & & $7.65,^{c} 7.66^{d}$ \\
\hline
\end{tabular}

sible candidates for the $\pi \rightarrow \pi^{*}$ excitation. They used their calculated adiabatic excitation energy, $6.82 \mathrm{eV}$, to propose the $\pi-\pi^{*}$ state assignment. This may be due to the fact that their calculated vertical excitation energy, $7.46 \mathrm{eV}$, of the same state is far away from the experimental values. However, Chang $^{2}$ raised doubts over this calculated adiabatic excitation energy of $\pi$ - $\pi^{*}$ state. ${ }^{1}$ He found that the $\pi$ - $\pi^{*}$ state has the lowest energy at $90^{\circ}$ (completely different from their first report), however, he neither mentioned the exact adiabatic energy nor did he make a reassignment for $\pi \rightarrow \pi^{*}$ excitation. According to that study, ${ }^{2}$ the adiabatic energy of $\pi-\pi^{*}$ state was around $5 \mathrm{eV}$ !

As mentioned earlier, the $\pi$ - $\pi^{*}$ vertical excitation energy obtained in the present study $(6.96 \mathrm{eV})$ agrees very well with the experimental value $(6.7-6.9 \mathrm{eV})$. Our results show that the oscillator strength is mainly carried by $\pi-\pi^{*}$ transition in the vertical region (Table III). These results clearly support the fact that the maximum in the absorption spectrum is due to the $\pi \rightarrow \pi^{*}$ excitation. However, the oscillator strengths obtained in the previous study using CASSCF level of theory for $\pi \rightarrow 3 s$ and $\pi \rightarrow \pi^{*}$ transitions are close to each other $(0.0515$ and 0.0818$){ }^{1}$

Walsh $^{39}$ and Merer and Mulliken ${ }^{40}$ analyzed the $\pi$ $\rightarrow \pi^{*}$ transition in ethylene and concluded that the $\pi-\pi^{*}$ state has an equilibrium geometry which is twisted $90^{\circ}$ about the $\mathrm{C}=\mathrm{C}$ bond with the $\mathrm{CH}_{2}$ groups deformed. Walsh ${ }^{39}$ pre-

TABLE II. Vinly chloride $\pi \rightarrow \pi^{*}$ vertical excitation energies $(\Delta E$ in $\mathrm{eV})$ using the SAC-CI method with various basis sets at experimental geometry (Ref. 38).

\begin{tabular}{|c|c|c|c|c|}
\hline \multirow[b]{2}{*}{ Method } & \multicolumn{2}{|c|}{ Basis set } & \multirow{2}{*}{$\begin{array}{l}\text { Rydberg function } \\
\text { (two sets of } s p d \text { ) }\end{array}$} & \multirow[b]{2}{*}{$\Delta E$} \\
\hline & $\mathrm{H}$ & $\mathrm{C}$ and $\mathrm{Cl}$ & & \\
\hline \multirow{9}{*}{ SAC-CI } & DZP & DZP & On $\mathrm{BC}$ and on $\mathrm{Cl}$ & 7.432 \\
\hline & cc-pVTZ & cc-pVTZ & On $\mathrm{BC}$ and on $\mathrm{Cl}$ & 7.211 \\
\hline & cc-pVTZ & aug-cc-pVTZ(-f) & On $\mathrm{BC}$ and on $\mathrm{Cl}$ & 7.116 \\
\hline & DZP & DZP & On $\mathrm{C}$ and $\mathrm{Cl}$ & 7.351 \\
\hline & cc-pVTZ & cc-pVTZ & On $\mathrm{C}$ and $\mathrm{Cl}$ & 7.155 \\
\hline & cc-pVTZ(-pd) & aug-cc-pVTZ(-f) & On $\mathrm{C}$ and $\mathrm{Cl}$ & 7.134 \\
\hline & cc-pVTZ(-pd) & aug-cc-pVTZ & On $\mathrm{C}$ and $\mathrm{Cl}$ & 7.065 \\
\hline & cc-pVTZ(-d) & aug-cc-pVTZ & On $\mathrm{C}$ and $\mathrm{Cl}$ & 7.028 \\
\hline & cc-pVTZ & aug-cc-pVTZ(-f) & On $\mathrm{C}$ and $\mathrm{Cl}$ & 7.068 \\
\hline Expt. & cc-pVTZ & aug-cc-pVTZ & On $\mathrm{C}$ and $\mathrm{Cl}$ & $\begin{array}{c}7.103 \\
6.70,^{\mathrm{a}, \mathrm{b}} 6.90,^{\mathrm{c}} \\
6.72,^{\mathrm{d}} 6.74^{\mathrm{e}}\end{array}$ \\
\hline $\begin{array}{l}\text { Reference } 4 . \\
\text { Reference } 8 . \\
\text { Reference } 10 \\
\text { Reference } 11 \\
\text { Reference } 12\end{array}$ & & & & \\
\hline
\end{tabular}


TABLE III. Several lowest-lying singlet excited states of $\mathrm{C}_{2} \mathrm{H}_{3} \mathrm{Cl}$ obtained using SAC-CI with cc-pVTZ(-d) for $\mathrm{H}$ and aug-cc-pVTZ for $\mathrm{C}$ and $\mathrm{Cl}$ at experimental geometry (Ref. 38). Two sets of Rydberg spd functions for both carbons and for chlorine were added. Excitation energies $(\Delta E)$, oscillator strengths $(f)$, and second moments $\left(\left\langle r^{2}\right\rangle\right)$. Note that the Rydberg $3 s, 3 p$, and $3 d$ orbitals are predominantly attributed to carbons while the Rydberg $4 s$ and $4 p$ orbitals are attributed to chlorine.

\begin{tabular}{|c|c|c|c|c|c|c|}
\hline \multirow[b]{2}{*}{ State } & \multirow[b]{2}{*}{ Nature } & \multicolumn{3}{|c|}{ SAC-CI } & \multicolumn{2}{|c|}{$\Delta E(\mathrm{eV})$} \\
\hline & & $\Delta E(\mathrm{eV})$ & $f$ & $\left\langle r^{2}\right\rangle$ & Other theory & Expt. \\
\hline $1{ }^{1} A^{\prime}$ & Ground state & & & 245.4 & & \\
\hline $1{ }^{1} A^{\prime \prime}$ & $\pi-3 s / \sigma_{\mathrm{C}-\mathrm{Cl}}^{*}$ & 6.81 & 0.0111 & 274.4 & $6.68^{\mathrm{a}}$ & \\
\hline $2{ }^{1} A^{\prime}$ & $\pi-\pi^{*}$ & 6.96 & 0.3274 & 257.1 & $\begin{array}{l}7.46^{\mathrm{a}} \\
7.40^{\mathrm{e}}\end{array}$ & $\begin{array}{l}6.7,{ }^{\mathrm{b}, \mathrm{c}} 6.9,^{\mathrm{d}} \\
6.72,{ }^{\mathrm{f}} 6.74^{\mathrm{g}}\end{array}$ \\
\hline $2{ }^{1} A^{\prime \prime}$ & $\pi-\sigma_{\mathrm{C}-\mathrm{Cl}}^{*} / 3 s$ & 6.99 & 0.0035 & 268.0 & $\begin{array}{l}7.27^{\mathrm{a}} \\
7.26^{\mathrm{e}}\end{array}$ & \\
\hline $3{ }^{1} A^{\prime \prime}$ & $\pi-3 p \sigma_{x}$ & 7.48 & 0.0006 & 297.6 & $7.48^{\mathrm{a}}$ & \\
\hline $4{ }^{1} A^{\prime \prime}$ & $\pi-3 p \sigma_{y}$ & 7.70 & 0.0102 & 312.6 & $7.56^{\mathrm{a}}$ & \\
\hline $5^{1} A^{\prime \prime}$ & $n_{\mathrm{Cl}} \pi^{*}$ & 7.82 & 0.0005 & 251.6 & $7.9^{\mathrm{e}}$ & \\
\hline $3{ }^{1} A^{\prime}$ & $\pi-3 p \pi$ & 7.89 & 0.0098 & 309.7 & & $7.817,{ }^{\mathrm{h}} 7.82^{\mathrm{i}}$ \\
\hline $4{ }^{1} A^{\prime}$ & $n_{\mathrm{Cl}^{-}} \sigma_{\mathrm{C}-\mathrm{Cl}}^{*}$ & 8.20 & 0.0012 & 256.2 & $8.50^{\mathrm{e}}$ & \\
\hline $6{ }^{1} A^{\prime \prime}$ & $\pi-3 d \sigma / 4 s$ & 8.42 & 0.0152 & 334.8 & & $8.33^{\mathrm{h}, \mathrm{i}}$ \\
\hline $7{ }^{1} A^{\prime \prime}$ & $\pi-3 d \sigma / 4 p \sigma_{x}$ & 8.46 & 0.0001 & 354.4 & & $8.38^{\mathrm{h}, \mathrm{i}}$ \\
\hline $5{ }^{1} A^{\prime}$ & $\pi-3 d \pi / 4 p \pi$ & 8.57 & 0.0050 & 362.2 & & $8.52^{\mathrm{h}}$ \\
\hline $6{ }^{1} A^{\prime}$ & $n_{\mathrm{Cl}^{-}}-3 s$ & 8.60 & 0.0465 & 293.0 & & $8.51^{\mathrm{h}, \mathrm{i}}$ \\
\hline
\end{tabular}

${ }^{\mathrm{a}}$ Reference 1 .

${ }^{\mathrm{b}}$ Reference 4.

${ }^{c}$ Reference 8 .

${ }^{\mathrm{d}}$ Reference 10.

${ }^{\mathrm{e}}$ Reference 6.

${ }^{\mathrm{f}}$ Reference 11

${ }^{\mathrm{g}}$ Reference 12.

${ }^{\mathrm{h}}$ Reference 15.

${ }^{\mathrm{i}}$ Reference 18.

dicted that the Raman spectrum of ethylene would show activity in the $\mathrm{C}=\mathrm{C}$ bond stretching mode, the $\mathrm{C}=\mathrm{C}$ twist, and various $\mathrm{CH}_{2}$ bends and deformations. These predictions were confirmed by Ziegler and Hudson ${ }^{41}$ and Sension and Hudson. ${ }^{33}$ Like ethylene, the emission spectra of $\mathrm{VC}$ obtained by Browning et al. ${ }^{16}$ also indicated $\mathrm{C}=\mathrm{C}$ stretching and twisting motion as well as $\mathrm{CH}_{2}$ bending or deformation, verifying that excitation has $\pi \rightarrow \pi^{*}$ character. All these facts support that the strong peak in the absorption spectrum is due to the $\pi \rightarrow \pi^{*}$ excitation.

Excitation energies obtained for other states also agree very well with the available experimental values (Table III). The $\pi-\sigma_{\mathrm{C}-\mathrm{Cl}}^{*}, n_{\mathrm{Cl}}-\pi^{*}$, and $n_{\mathrm{Cl}}-\sigma_{\mathrm{C}-\mathrm{Cl}}^{*}$ states are assigned at $6.99,7.82$, and $8.20 \mathrm{eV}$, respectively.

We also investigated a few low-lying triplet states by using SAC-CI with the same basis set used to study the singlet states. It is worth to mention here that the vertical excitation energy of $4.12 \mathrm{eV}$ obtained for ${ }^{3}\left(\pi-\pi^{*}\right)$ state is very close to the experimental value, $4.13 \mathrm{eV},{ }^{12}$ whereas it is $4.25 \mathrm{eV}$ in MRCI calculation. ${ }^{2}$ The detailed results regarding excitations to the triplet states will be published in a forthcoming article.

Chang $^{2}$ studied the potential-energy surface of the first excited state of VC by using CASSCF. It showed that departing from the planar equilibrium structure through a barrier, the first excited state is mainly of $\pi-\sigma_{\mathrm{C}-\mathrm{Cl}}^{*}$ character as the $\mathrm{C}-\mathrm{Cl}$ distance increases, whereas it is of $\pi-\pi^{*}$ character as the twisting angle increases. This is as a result of the forma- tion of avoided crossings between $\pi-3 s$ which has been assigned as the bright state by Chang and $\mathrm{Chen}^{1}$ and $\pi-\sigma_{\mathrm{C}-\mathrm{Cl}}^{*}$ states at planar geometries (both have the same $A^{\prime \prime}$ symmetry) and between $\pi-3 s$ and $\pi-\pi^{*}$ states at nonplanar structures (all states have the same $A$ symmetry). Accordingly, $\mathrm{Chang}^{2}$ concluded that the dissociation of $\mathrm{Cl}$ takes place via two pathways: one is through $\pi-\sigma_{\mathrm{C}-\mathrm{Cl}}^{*}$ at planar leading to fast $\mathrm{Cl}$ and the other through $\pi-\pi^{*}$ at twisted geometries from which internal conversion to the ground state and subsequent dissociation produces slow $\mathrm{Cl}$.

Our results, however, show some interesting differences. The PES of some excited states along the $\mathrm{C}-\mathrm{Cl}$ bond length and $\mathrm{C}=\mathrm{C}$ bond twisting were depicted in Figs. 1 and 2, respectively. For this purpose, single point calculations on different $\mathrm{C}-\mathrm{Cl}$ bond lengths and twisting angles were performed at SAC-CI/d95 $(d, p)$ level with all other internal coordinates were kept at the ground-state values optimized at SAC/d95(d) level. The orbital characters given in Table IV show that $\sigma_{\mathrm{C}-\mathrm{Cl}}^{*}$ has an antibonding nature along $\mathrm{C}-\mathrm{Cl}$ bond. The nature of the $\pi-\sigma_{\mathrm{C}-\mathrm{Cl}}^{*}$ state was in debate in literature ${ }^{5,6}$ as mentioned in the Introduction section. The present study clearly shows that this state is a repulsive state as shown in Fig. 1(a).

The PES along the $\mathrm{C}-\mathrm{Cl}$ bond length clearly reveals the formation of an avoided crossing between the bright $\pi$ - $\pi^{*}$ and $n_{\mathrm{Cl}} \sigma_{\mathrm{C}-\mathrm{Cl}}^{*}$ states (both have the same symmetry $A^{\prime}$ ) at around $1.85 \AA$. This reveals that the excited VC transfers 


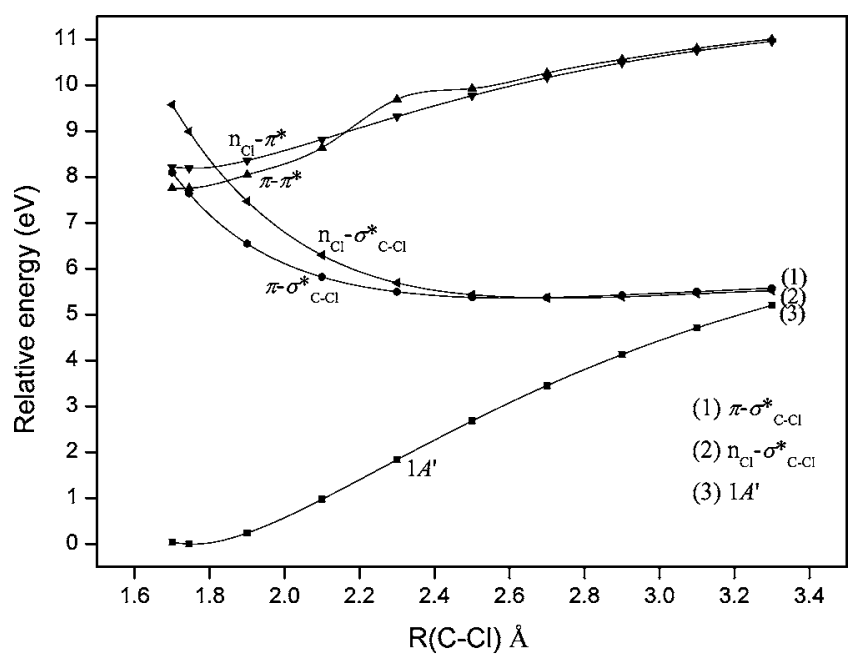

(a)

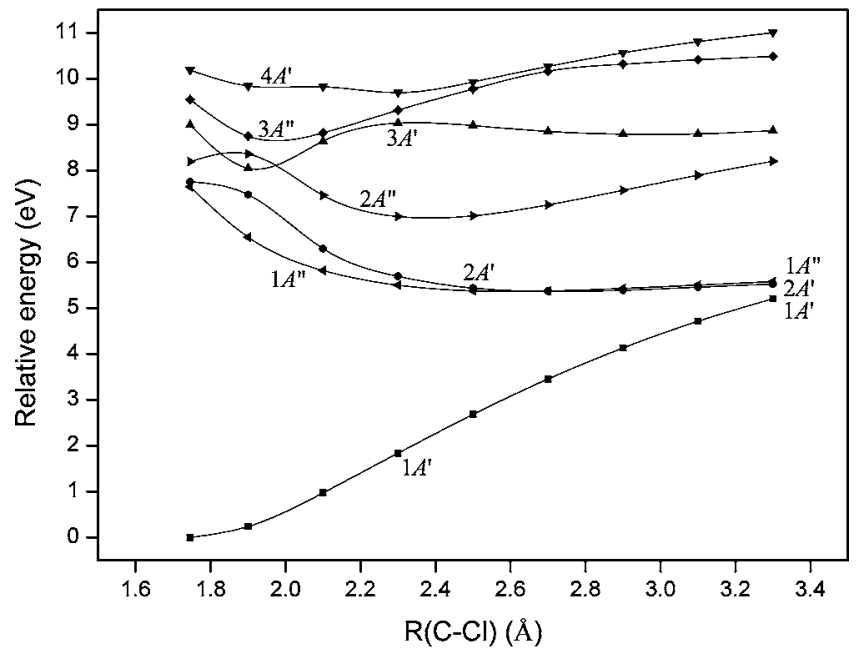

(b)

FIG. 1. PES along the $\mathrm{C}-\mathrm{Cl}$ bond with the other geometrical parameters optimized for the ground state: (a) diabatic PES of $\pi-\sigma_{\mathrm{C}-\mathrm{Cl}}^{*}, \pi-\pi^{*}, n_{\mathrm{Cl}} \pi^{*}$, and $n_{\mathrm{Cl}} \sigma_{\mathrm{C}-\mathrm{Cl}}^{*}$ states and (b) adiabatic PES of several low-lying excited states.

from its bound $\pi-\pi^{*}$ state to the dissociative $n_{\mathrm{Cl}} \sigma_{\mathrm{C}-\mathrm{Cl}}^{*}$ state through level crossing. So dissociation can take place through this repulsive $n_{\mathrm{Cl}} \sigma_{\mathrm{C}-\mathrm{Cl}}^{*}$ state.

The PES along torsion shows that the low-lying two states $\pi-\sigma_{\mathrm{C}-\mathrm{Cl}}^{*}$ and $\pi-\pi^{*}$ start mixing from the very beginning of torsion. As the twisting angle increases, the mixing becomes stronger. As a result, the $\pi-\pi^{*}$ character of the first excited state increases and simultaneously the $\pi-\sigma_{\mathrm{C}-\mathrm{Cl}}^{*}$ character of the second and third states dominates at higher twisting angles. It reflects the bound nature of the $\pi-\sigma_{\mathrm{C}-\mathrm{Cl}}^{*}$ state and the dissociation of $\mathrm{VC}$ could occur through the well of $\pi-\pi^{*}$ state or through the ground state followed by internal conversion from $\pi-\pi^{*}$ to the ground state. However, the mixing of the $\pi-\pi^{*}$ and $\pi-\sigma_{\mathrm{C}-\mathrm{Cl}}^{*}$ states due to twisting could possibly lead into fast dissociation of chlorine atoms on $\pi-\sigma_{\mathrm{C}-\mathrm{Cl}}^{*}$ state which has a repulsive nature along $\mathrm{C}-\mathrm{Cl}$ bond coordinate. So there is a possibility for the $\mathrm{C}-\mathrm{Cl}$ bond dissociation through $\pi-\sigma_{\mathrm{C}-\mathrm{Cl}}^{*}$ state in the $\mathrm{C}-\mathrm{Cl}$ bond coordinate after it started mixing with $\pi-\pi^{*}$ state.

Earlier experimental studies ${ }^{6,42}$ on VC concluded that the

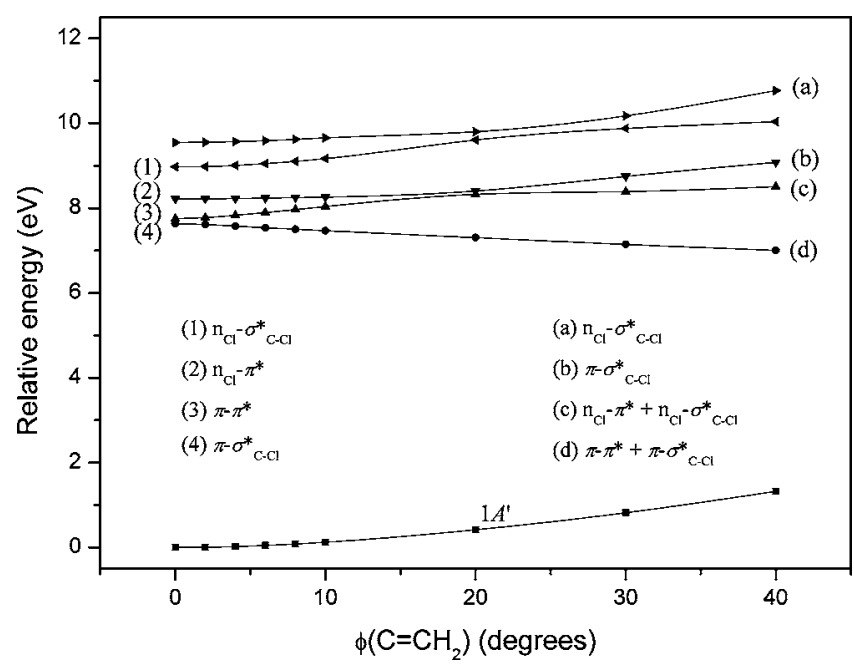

FIG. 2. PES of several low-lying excited states along $\mathrm{C}=\mathrm{C}$ twisting with the other geometrical parameters optimized for the ground state.

dissociation from the excited state is much faster $(40 \pm 10 \mathrm{fs})$ than from the ground state $(110 \mathrm{ps})$. That means the fast dissociation of chlorine atoms resulted from the repulsive excited state and not from the ground state followed by twisting and internal conversion. So it is obvious that the dissociation from the ground state has less importance.

The forgoing facts reveal the following: The main excitation is the $\pi \rightarrow \pi^{*}$ transition and at planar geometries along $\mathrm{C}-\mathrm{Cl}$ bond coordinate, the bright $\pi$ - $\pi^{*}$ state forms an avoided crossing with the $n_{\mathrm{Cl}}-\sigma_{\mathrm{C}-\mathrm{Cl}}^{*}$ state which leads to fast $\mathrm{C}-\mathrm{Cl}$ bond dissociation. Twisting causes mixing of $\pi-\pi^{*}$ state with $\pi-\sigma_{\mathrm{C}-\mathrm{Cl}}^{*}$ state, however, dissociation from the $\pi-\sigma_{\mathrm{C}-\mathrm{Cl}}^{*}$ state after initial twisting may not have much importance because the fast dissociation takes place at the planar structure and the dissociation takes place within a time much shorter than molecular twisting. ${ }^{6,7,42}$ But there may be a small possibility for this $\pi-\sigma_{\mathrm{C}-\mathrm{Cl}}^{*}$ state to be involved in the $\mathrm{C}-\mathrm{Cl}$ dissociation. There is a small transition from the ground state to the Rydberg $3 s$ state (see Table III) in the excitation. This state, $\pi-3 s$, could form an avoided crossing with the dissociative $\pi$ - $\sigma_{\mathrm{C}-\mathrm{Cl}}^{*}$ state in the planar structure (both have the same $A^{\prime \prime}$ symmetry), as mentioned by Chang, ${ }^{1}$ which could lead to fast dissociation like that from the repulsive $n_{\mathrm{Cl}}-\sigma_{\mathrm{C}-\mathrm{Cl}}^{*}$ state. However, the oscillator strength for $\pi$ $\rightarrow 3 s$ transition is very small compared with that for $\pi$ $\rightarrow \pi^{*}$ transition and hence it is obvious that the dissociation through this $\pi-\sigma_{\mathrm{C}-\mathrm{Cl}}^{*}$ state has a minor contribution. Again, the $\mathrm{C}-\mathrm{Cl}$ bond dissociation through the ground state does not have much importance. All these facts suggest that the fast dissociation of the $\mathrm{C}-\mathrm{Cl}$ bond takes places mainly through the repulsive $n_{\mathrm{Cl}} \sigma_{\mathrm{C}-\mathrm{Cl}}^{*}$ state.

TABLE IV. Nature of $\pi, \pi^{*}, \sigma_{\mathrm{C}-\mathrm{Cl}}^{*}$, and $n_{\mathrm{Cl}}$ orbitals. (d) Dominant.

\begin{tabular}{ccc}
\hline \hline Nature & $\mathrm{C}=\mathrm{C}$ & $\mathrm{C}-\mathrm{Cl}$ \\
\hline$\pi$ & Bonding (d) & Antibonding \\
$\pi^{*}$ & Antibonding (d) & Antibonding \\
$\sigma_{\mathrm{C}-\mathrm{Cl}}^{*}$ & Antibonding & Antibonding (d) \\
$n_{\mathrm{Cl}}$ & Antibonding & Bonding (d) \\
\hline \hline
\end{tabular}


Chang ${ }^{2}$ studied the first singlet excited state to understand the dissociation process, since presumably the dissociation could take place through this state. It should be mentioned here that our results show that the first two states $\pi-\sigma_{\mathrm{C}-\mathrm{Cl}}^{*}$ and $n_{\mathrm{Cl}}-\sigma_{\mathrm{C}-\mathrm{Cl}}^{*}$ are energetically very close once the $\mathrm{C}-\mathrm{Cl}$ bond length reaches at around $2.4 \AA$ and, in fact, the $n_{\mathrm{Cl}}-\sigma_{\mathrm{C}-\mathrm{Cl}}^{*}$ state becomes the first excited state when the $\mathrm{C}-\mathrm{Cl}$ bond length increases to around $3 \AA$ (see Fig. 1).

The present PES calculation does not include Rydberg states; however, we believe that the inclusion of Rydberg states in the PES will not have any adverse effect beyond the Frank-Condon region and we expect that the $\mathrm{C}-\mathrm{Cl}$ bond dissociation would occur on valence states. Results of excitations to the higher Rydberg states and the detailed twodimensional (2D) PES of low-lying excited states, including Rydberg states, incorporating both $\mathrm{C}-\mathrm{Cl}$ bond length and $\mathrm{C}=\mathrm{C}$ twisting at the excited-state geometries will be presented in a future publication.

\section{CONCLUDING REMARKS}

The vertical excitation energies obtained using SAC-CI with the necessary basis sets agree very well with the experimental values. The present study not only clarified the existing ambiguity in the assignment for the optically bright state of $\mathrm{VC}$ but also gave the confidence that the same level of theory could be used to obtain the theoretical electronic spectra of other chlorinated ethylenes, which would be useful to assign the observed spectra of these important atmospheric pollutants.

In the case of photodissociation, the optically bright $\pi-\pi^{*}$ state forms an avoided crossing with $n_{\mathrm{Cl}} \sigma_{\mathrm{C}-\mathrm{Cl}}^{*}$ state which leads to fast $\mathrm{C}-\mathrm{Cl}$ bond dissociation. Though $\pi-\sigma_{\mathrm{C}-\mathrm{Cl}}^{*}$ is a repulsive state, its participation in the dissociation is expected to be small.

\section{ACKNOWLEDGMENT}

This study has been supported by the Grant for Creative Scientific Research from the Ministry of Education, Science, Sports, and Culture of Japan.

${ }^{1}$ J.-L. Chang and Y.-T. Chen, J. Chem. Phys. 116, 7518 (2002).

${ }^{2}$ J.-L. Chang, J. Chem. Phys. 122, 194321 (2005).

${ }^{3}$ M. B. Robin, Higher Excited States of Polyatomic Molecules (Academic, New York, 1975), Vol. 2, p. 56.

${ }^{4}$ M. J. Berry, J. Chem. Phys. 61, 3114 (1974).

${ }^{5}$ M. Umemoto, K. Seki, H. Shinohara, U. Nagashima, N. Nishi, M. Kinoshita, and R. Shimada, J. Chem. Phys. 83, 1657 (1985).
${ }^{6}$ K. Tonokura, L. B. Daniels, T. Suzuki, and K. Yamashita, J. Phys. Chem. A 101, 7754 (1997).

${ }^{7}$ D. A. Blank, W. Sun, A. G. Suits, Y. T. Lee, S. W. North, and G. E. Hall, J. Chem. Phys. 108, 5414 (1998).

${ }^{8}$ A. D. Walsh, Trans. Faraday Soc. 41, 35 (1945).

${ }^{9}$ S. P. Sood and K. Watanabe, J. Chem. Phys. 45, 2913 (1966).

${ }^{10}$ J. H. Moore, Jr., J. Phys. Chem. 76, 1130 (1972).

${ }^{11}$ A. Kuppermann, W. M. Flicker, and O. A. Mosher, Chem. Rev. (Washington, D.C.) 79, 77 (1979).

${ }^{12}$ C. F. Koerting, K. N. Walzl, and A. Kuppermann, Chem. Phys. Lett. 109, 140 (1984).

${ }^{13}$ K. H. Sze, C. E. Brion, A. Katrib, and B. El-Issa, Chem. Phys. 137, 369 (1989).

${ }^{14}$ R. Locht, B. Leyh, K. Hottmann, and H. Baumgartel, Chem. Phys. 220, 207 (1997).

${ }^{15}$ B. A. Williams and T. A. Cool, J. Phys. Chem. 97, 1270 (1993).

${ }^{16}$ P. W. Browning, D. C. Kitchen, M. F. Arendt, and L. J. Butler, J. Phys. Chem. 100, 7765 (1996).

${ }^{17}$ J.-L. Chang, J.-C. Shieh, J.-C. Wu, R. Li, and Y.-T. Chen, Chem. Phys. Lett. 325, 369 (2000).

${ }^{18}$ J.-L. Chang, R. Li, J.-C. Wu, J.-C. Shieh, and Y.-T. Chen, J. Chem. Phys. 115, 5925 (2001).

${ }^{19}$ A. Fahr and A. H. Laufer, J. Phys. Chem. 89, 2906 (1985).

${ }^{20}$ Y. Mo, K. Tonokura, Y. Matsumi et al., J. Chem. Phys. 97, 4815 (1992).

${ }^{21}$ Y. Huang, Y.-A. Yang, G.-X. He, and R. J. Gordon, J. Chem. Phys. 99, 2752 (1993).

${ }^{22}$ Y. Huang, Y.-A. Yang, G. He, S. Hashimoto, and R. J. Gordon, J. Chem. Phys. 103, 5476 (1995).

${ }^{23}$ T. L. Myers, D. C. Kitchen, B. Hu, and L. J. Butler, J. Chem. Phys. 104, 5446 (1996).

${ }^{24}$ K. Sato, S. Tsunashima, T. Takayanagi, G. Fujisawa, and A. Yokoyama, J. Chem. Phys. 106, 10123 (1997)

${ }^{25}$ H. Nakatsuji and K. Hirao, J. Chem. Phys. 68, 2053 (1978).

${ }^{26}$ H. Nakatsuji, Chem. Phys. Lett. 59, 362 (1978).

${ }^{27}$ H. Nakatsuji, Chem. Phys. Lett. 67, 329 (1979); 67, 334 (1979).

${ }^{28}$ H. Nakatsuji, Acta Chim. Hung. 129, 719 (1992); Computational Chemistry-Review of Current Trends (World Scientific, Singapore, 1997), Vol. 2, pp. 62-124

${ }^{29}$ M. J. Frisch, G. W. Trucks, H. B. Schlegel et al., GaUSSIAN 03, Revision B.01, Gaussian, Inc., Pittsburgh, PA, 2003.

${ }^{30}$ S. Huzinaga, J. Chem. Phys. 42, 1293 (1965); T. H. Dunning, Jr., ibid. 53, 2823 (1970).

${ }^{31}$ T. H. Dunning, Jr., J. Chem. Phys. 90, 1007 (1989); D. E. Woon and T. H. Durning, Jr., ibid. 98, 1358 (1993).

${ }^{32}$ A. J. Merer and R. S. Mulliken, Chem. Rev. (Washington, D.C.) 69, 639 (1969); R. S. Mulliken, J. Chem. Phys. 66, 2448 (1977); 71, 556 (1979).

${ }^{33}$ R. J. Sension and B. S. Hudson, J. Chem. Phys. 90, 1377 (1989).

${ }^{34}$ R. Lindh and B. O. Roos, Int. J. Quantum Chem. 35, 813 (1989).

${ }^{35}$ J. D. Watts, S. R. Gwaltney, and R. J. Bartlett, J. Chem. Phys. 105, 6979 (1996).

${ }^{36}$ S. Krebs and R. J. Buenker, J. Chem. Phys. 106, 7208 (1997).

${ }^{37}$ E. Hirota, Y. Endo, S. Saito, K. Yoshida, I. Yamaguchi, and K. Machida, J. Mol. Spectrosc. 89, 223 (1981).

${ }^{38}$ I. Merke, L. Poteau, G. Wlodarczak, A. Bouddou, and J. Demaison, J. Mol. Spectrosc. 177, 232 (1996).

${ }^{39}$ A. D. Walsh, J. Chem. Soc. 1953, 2353.

${ }^{40}$ A. J. Merer and R. S. Mulliken, J. Chem. Phys. 50, 1026 (1969).

${ }^{41}$ L. D. Ziegler and B. S. Hudson, J. Chem. Phys. 79, 1197 (1983).

${ }^{42}$ P. Farmanara, V. Stert, and W. Radloff, Chem. Phys. Lett. 288, 518 (1998). 